\title{
Tensor correlation, pairing interaction and deformation in Ne isotopes and Ne hypernuclei
}

\author{
A. $\mathrm{Li}^{1 *}$, E. Hiyama ${ }^{1 \dagger}$, X.-R. Zhou ${ }^{2 \ddagger}$, H.Sagawa ${ }^{1,3 \S}$ \\ ${ }^{1}$ RIKEN Nishina Center, RIKEN, Wako 351-0198, Japan \\ 2 Department of Physics and Institute of Theoretical Physics and Astrophysics, \\ Xiamen University, Xiamen 361005, China \\ ${ }^{3}$ Center for Mathematics and Physics, University of Aizu, \\ Aizu-Wakamatsu, Fukushima 965-8560, Japan
}

(Dated: August 18, 2018)

\begin{abstract}
We study tensor and pairing effects on the quadruple deformation of neon isotopes based on a deformed Skyrme-Hartree-Fock model with BCS approximation for the pairing channel. We extend the SkyrmeHartree-Fock formalism for the description of single- and double-lambda hypernuclei adopting two different hyperon-nucleon interactions. It is found that the interplay of pairing and tensor interactions is crucial to derive the deformations in several neon isotopes. Especially, the shapes of ${ }^{26,30} \mathrm{Ne}$ are studied in details in comparisons with experimentally observed shapes. Furthermore the deformations of the hypernuclei are compared with the corresponding neon isotopic cores in the presence of tensor force. We find the same shapes with somewhat smaller deformations for single $\Lambda$-hypernuclei compared with their core deformations. It is also pointed out that the latest version of hyperon interaction, the ESC08b model, having a deeper $\Lambda$ potential makes smaller deformations for hypernuclei than those of another NSC97f model.
\end{abstract}

PACS numbers: 21.10.Dr, 21.30.Fe, 21.80.+a

\footnotetext{
* liang@ riken.jp

† hiyama@ riken.jp

‡xrzhou@xmu.edu.cn

§sagawa@u-aizu.ac.jp
} 


\section{INTRODUCTION}

Recently, several neutron-rich neon isotopes are confirmed to be deformed by the observation of the low excitation energies and $B(E 2)$ values of the first excited $2^{+}$states [1-6], including ${ }^{30} \mathrm{Ne}$ with a $N=20$ magic number. These empirical evidences become a strong motivation to make extensive experimental and theoretical studies on the neon isotope [7-12]. For example, experimentally, Takechi et al. [7] succeeded in measuring the interaction cross section $\sigma_{\mathrm{I}}$ of ${ }^{20-32} \mathrm{Ne}$ isotopes and as a result, they have found that starting mass number 25, the $\sigma_{\mathrm{I}}$ data exceed the systematic mass-number dependence of $\sigma_{\mathrm{I}}$ for stable nuclei. These observations indicate possible large nuclear deformations for these nuclei. Particularly, the much enhanced $\sigma_{\mathrm{I}}$ data suggest that there are deformed halos for ${ }^{29} \mathrm{Ne}$ and ${ }^{31} \mathrm{Ne}$. The halo structure of ${ }^{31} \mathrm{Ne}$ has been clearly observed also by the measurement of Coulomb breakup cross section by Nakamura et al. [8]. Urata et al. [9] then studied in details this ${ }^{31} \mathrm{Ne}$ halo structure in the framework of deformed ${ }^{30} \mathrm{Ne}+\mathrm{n}$ two-body model. Moreover, two of the author in the present work, H. S. and X. Z. [10] studied the neon isotope within the deformed Skyrme-Hartree-Fock (SHF) model with two Skyrme interactions SGII and SIII, and investigated the neutron number dependence of deformation properties along the chain of neon isotopes. Reaction calculations of ${ }^{20-32} \mathrm{Ne}$ isotopes by the antisymmetrized molecular dynamics (AMD) have also been extensively done [11, 12], and they suggested large deformations for most of the neutron-rich isotopes, consistent with the indication of recent experiments.

However, some of the experimentally-determined shapes of isotopic nuclei are not reproduced theoretically by many mean-field models. To take ${ }^{30} \mathrm{Ne}$ as an example, the observed low excitation energy of $2_{1}^{+}$state indicates a large deformation for this nuclei, but both the HartreeFock-Bogoliubov (HFB) theory [13] and the relativistic HFB (RHFB) theory [14] give a spherical result, as well as the above-mentioned SHF model [10]. Since the nuclear shape is closely related to its shell structure, a tensor-force-driven deformation has been proposed recently [15-17] in the occurrence of large oblate deformation in ${ }^{42} \mathrm{Si}$. Because the monopole interaction of tensor force [18, 19] will result in a smaller $1 s 1 / 2-0 d 5 / 2$ proton gap when more neutrons occupy $0 f 7 / 2$ state, the nucleus favors energetically to be in an oblately-deformed state. This indicates the crucial role of the tensor force on the deformation of neutron-rich nuclei, even $N=28$ is a magic number and supposed to favor a spherical shape.

Many Skyrme parameter sets which have been widely used do not include the tensor contribution, although it was suggested more than 50 years ago by Skyrme [20]. Only recently, a Skyrme interaction which includes the tensor contribution was proposed [21], and Colò et al. [22] and Brink \& Stancu [23] have added Skyrme-type tensor force on top of the existing standard parameterizations SLy5 and SIII, respectively. After that, Lesinski et al. [24] built a set of 36 effective interactions with a systematical adjusting of the tensor coupling strengths. Each of these parameterizations has been fitted to give a reasonable description for ground states of finite nuclei such as binding energies and radii. The inclusion of tensor terms in the calculations achieved considerable success in explaining various nuclear structure problems [22-36]. The present work is devoted to a systematic study of the tensor effect on the deformation of neon isotopes, and theoretical results will be confronted directly with recent experiments.

In addition, lately, the pairing interaction between nucleons has been extended to be isospindependent [37, 38], and global SHF calculations [39] with an isospin-dependent contact pairing interaction are shown to have a better agreement with empirical data than the results without isospindependence. This may be a hint that the pairing strength without the isospin dependence, incorporated in the previous SHF study, should be reexamined taking care of the isospin-dependence 
nature. This is because some neutron-rich nuclei far from the line of stability have large isospins, and the pairing strength might be largely reduced. It is also known that the evolution of the deformation is rather model-dependent especially on the role of the pairing interaction [11-14]. Therefore as a first step it is of great interest to investigate how the change of pairing strength will influence the shape of nuclei. We will show in this study that the pairing interaction has a decisive effect on the nuclear deformation of several neutron-rich nuclei.

In hypernuclear physics, it is one of the interesting subjects to study the modification of nuclear structure when a hyperon such as $\Lambda$ particle is added to a nucleus. Theoretically, Motoba et al. [40] pointed out that sizes of $p$-shell $\Lambda$ hypernuclei are shrunk by the addition of a $\Lambda$ particle in comparison with those of the core nuclei (It is called a gluelike role of the $\Lambda$ particle). They suggested that the sizable shrinkage effect was seen in the value of $B(E 2)$. Afterwards, the shrinkage effect on $B(E 2)$ values was studied in the case of $5 / 2^{+} \rightarrow 1 / 2^{+}$transition in ${ }_{\Lambda}^{7} \mathrm{Li}$ [41]. Then, the KEK-E419 experiment was successfully performed to measure this $B(E 2)$ and confirmed the shrinkage effect of $\Lambda$ particle on the nuclear size for the first time [42]. Also, some authors have studied the change of structure in heavier hypernuclei by addition of a $\Lambda$ particle [43-47]. Within the framework of mean-field models [43-45], they suggested the changes of deformation between core nuclei in ${ }^{12} \mathrm{C}$ and ${ }^{28,30,32} \mathrm{Si}$, and the corresponding hypernuclei by the addition of a $\Lambda$ particle. Furthermore it was pointed out from the study of ${ }_{\Lambda}^{9} \mathrm{Be}$ and ${ }_{\Lambda}^{13} \mathrm{C}$ by the AMD [47] that if a nucleus occupies a $1 p$ shell orbital, a hyperon may enhance the nuclear deformation.

It should be noted that in hypernuclear physics there have been a lot of ambiguities in proposed hyperon-nucleon $(Y N)$ interactions due to the limitation of $Y N$ scattering data. For a decade, by combining the $\gamma$-ray experimental data and theoretical calculations such as the shell model [48] and the clustering approach [49, 50], they succeeded in extracting information on its spin-dependent parts of $\Lambda N$ interaction. As a result, the most updated $Y N$ interaction, Nijmegen soft core potential such as the ESC08b model [51] is proposed. In the present work, we use this ESC08b potential to study the structure changes of the Ne isotopes. In addition, we use the NSC97f potential [52] which reproduces the observed binding energies of light $\Lambda$ hypernuclei. The main aim of this paper is to study the importance of tensor and pairing effects on the deformation properties of neon isotopes and the corresponding hypernuclei.

The paper is organized as follows. In Sec. II, we outline the necessary formalism. The numerical results and discussions are given in Sec. III. Finally, Sec. IV contains the main conclusions and future perspectives of this work.

\section{FORMALISM}

Our study is based on the deformed SHF method of Ref. [53], accompanied by a densitydependent contact pairing using the BCS approximation. An extended model was proposed for the description of hypernuclei in Refs. [43, 54] by including an effective microscopic lambda-nucleon interaction derived from Brueckner-Hartree-Fock (BHF) calculations of isospin-asymmetric nuclear matter using realistic Nijmegen YN potential [55]. In the following we outline some necessary formalism.

The total binding energy of a nucleus can be obtained self-consistently from the energy functional [24, 56]:

$$
\mathscr{E}_{N}=\mathscr{E}_{\mathrm{kin}}+\mathscr{E}_{\mathrm{Sk}}+\mathscr{E}_{\text {pair }}+\mathscr{E}_{\mathrm{Coul}}+\mathscr{E}_{\mathrm{corr}}
$$

where $\mathscr{E}_{\text {kin }}$ is the kinetic energy functional, $\mathscr{E}_{\mathrm{Sk}}$ is the Skyrme functional, $\mathscr{E}_{\text {pair }}$ is the pairing energy functional, $\mathscr{E}_{\mathrm{Coul}}$ is the Coulomb energy functional and $\mathscr{E}_{\text {corr }}$ is the center-of-mass correction. More 
TABLE I: Coupling strengths (in MeV) of various parameter sets used in the work.

\begin{tabular}{cccccccccccc}
\hline \hline & T14 & T24 & T34 & T44 & T54 & T61 & T62 & T63 & T64 & T65 & T66 \\
\hline$\alpha$ & 120 & 120 & 120 & 120 & 120 & -60 & 0 & 60 & 120 & 180 & 240 \\
\hline$\beta$ & -60 & 0 & 60 & 120 & 180 & 240 & 240 & 240 & 240 & 240 & 240 \\
\hline$\alpha_{T}$ & 38.5 & 24.7 & 12.8 & 8.97 & -3.48 & -200 & -131 & -80.5 & -0.246 & 49.9 & 113 \\
\hline$\beta_{T}$ & -15.2 & 19.4 & 57.8 & 113 & 150 & 178 & 196 & 177 & 218 & 196 & 204 \\
\hline$U$ & 92.5 & 59.2 & 30.8 & 21.5 & -8.36 & -480 & -314 & -193 & -0.591 & 120 & 271 \\
\hline$T$ & -165 & 33.7 & 247 & 521 & 727 & 1044 & 1256 & 1335 & 1047 & 823 & 709 \\
\hline \hline
\end{tabular}

physics details can be found in Refs. [24, 56] and references therein, and we will only address some for $\mathscr{E}_{\text {Skyrme }}$ and $\mathscr{E}_{\text {pair }}$, which are relevant for the present study.

To generate the Skyrme energy functional $\mathscr{E}_{\mathrm{Sk}}$, an effective zero-range two-body tensor force [20] is included in recent Skyrme parameterizations [21-24] as follows:

$$
\begin{aligned}
v_{T}(\mathbf{r})=\frac{T}{2}\{[ & \left.\left(\sigma_{1} \cdot \mathbf{k}^{\prime}\right)\left(\sigma_{2} \cdot \mathbf{k}^{\prime}\right)-\frac{1}{3}\left(\sigma_{1} \cdot \sigma_{2}\right) \mathbf{k}^{\prime}\right] \delta\left(\mathbf{r}_{1}-\mathbf{r}_{2}\right) \\
& \left.+\delta\left(\mathbf{r}_{1}-\mathbf{r}_{2}\right)\left[\left(\sigma_{1} \cdot \mathbf{k}\right)\left(\sigma_{2} \cdot \mathbf{k}\right)-\frac{1}{3}\left(\sigma_{1} \cdot \sigma_{2}\right) \mathbf{k}^{2}\right]\right\} \\
+ & U\left(\sigma_{1} \cdot \mathbf{k}^{\prime}\right) \boldsymbol{\delta}\left(\mathbf{r}_{1}-\mathbf{r}_{2}\right)\left(\sigma_{1} \cdot \mathbf{k}\right) \\
& \left.-\frac{1}{3}\left(\sigma_{1} \cdot \sigma_{2}\right)\left[\mathbf{k}^{\prime} \cdot \boldsymbol{\delta}\left(\mathbf{r}_{1}-\mathbf{r}_{2}\right)\right] \mathbf{k}\right\}
\end{aligned}
$$

where $T$ and $U$ are the coupling constants denoting the strength of the triplet-even and tripletodd tensor interaction, respectively. The operator $\mathbf{k} \equiv\left(\nabla_{1}-\nabla_{2}\right) / 2 i$ acts on the right, and $\mathbf{k}^{\prime} \equiv$ $-\left(\nabla_{1}-\nabla_{2}\right) / 2 i$ acts on the left. We will select several effective interactions with tensor terms included from Ref. [24] in the present study, to compare not only the cases with or without tensor terms, but also the cases with different tensor strengths.

In the Skyrme energy functional $\mathscr{E}_{\mathrm{Sk}}=\int \mathrm{d}^{3} r \mathscr{H}^{\mathrm{Sk}}(\mathbf{r})$, the tensor part contributes to the energy density $\mathscr{H}^{\mathrm{Sk}}$ in a combined way with the exchange term of central part as

$$
\begin{aligned}
\mathscr{H}_{T}^{\mathrm{Sk}} & =\frac{1}{2} \alpha\left(J_{n}^{2}+J_{p}^{2}\right)+\beta \vec{J}_{n} \cdot \vec{J}_{p} ; \\
\alpha & =\alpha_{C}+\alpha_{T} ; \quad \beta=\beta_{C}+\beta_{T} \\
\alpha_{C} & =\frac{1}{8}\left(t_{1}-t_{2}\right)-\frac{1}{8}\left(t_{1} x_{1}+t_{2} x_{2}\right) ; \\
\beta_{C} & =-\frac{1}{8}\left(t_{1} x_{1}+t_{2} x_{2}\right) \\
\alpha_{T} & =\frac{5}{12} U ; \quad \beta_{T}=\frac{5}{24}(T+U) .
\end{aligned}
$$

indicated by a subscript of $T$ or $C$, respectively. $\alpha$ represents the strength of like-particle coupling between neutron-neutron or proton-proton, and $\beta$ is that of the neutron-proton coupling. The coupling strengths of various parameter sets used in this study are collected in Tab. 1.

The pairing energy functional $\mathscr{E}_{\text {pair }}$ is introduced as:

$$
\mathscr{E}_{\text {pair }}=\frac{1}{4} \sum_{q \in\{p, n\}} \int \mathrm{d}^{3} r \chi_{q}^{*}(\mathbf{r}) \chi_{q}(\mathbf{r}) G(\mathbf{r})
$$


where $\chi(\mathbf{r})$ is the local pairing density matrix (addressed later), and $G(\mathbf{r})$ denotes the pairing strength function. We choose $G(\mathbf{r})=V_{\text {pair }}\left(1-\frac{\rho(\mathbf{r})}{\rho_{0}}\right)$ with $\rho_{0}=0.16 \mathrm{fm}^{-3}$, which corresponds to a density-dependent delta force for the pairing interaction. Based on the empirical neutron pairing gaps extracted by using the three-point mass difference formula [57] and the experimental binding energies of Ref. [58], we choose $V_{\text {pair }}=900 \mathrm{MeV}$, which can reproduce reasonably the gap data for the whole isotopic chain. This value is referred in the following as the full pairing cases (labeled as $\left.V_{\text {pair }}^{\text {full }}\right)$.

The local part of the pair density matrix $\chi(\mathbf{r})$ is written as

$$
\chi_{q}(\mathbf{r})=-2 \sum_{\substack{k \in \Omega_{q} \\ k>0}} f_{k} u_{k} v_{k}\left|\phi_{k}(\mathbf{r})\right|^{2}
$$

with $q \in\{p, n\}$, and $\Omega_{q}$ is the configuration space adapted. The $\phi_{k}$ are the singe particle (s.p.) wave functions and $v_{k}, u_{k}=\sqrt{1-v_{k}^{2}}$ are the pairing amplitudes. The cutoff factors $f_{k}$ are included to prevent the unrealistic pairing of highly excited states based on the employed pairing energy functional of Eq. (7), and more details are referred to Ref. [59].

From the nuclear energy functional $\mathscr{E}_{N}$ of Eq. (1), one can obtain the mean-field $\mathrm{HF}+\mathrm{BCS}$ equations for nucleons by standard functional derivative techniques, and they are applied to calculate the ground-state properties of nuclei. For the calculations of hypernuclei, the contribution due to the presence of hyperons should be included accordingly:

$$
\begin{aligned}
\mathscr{E} & =\mathscr{E}_{N}+\mathscr{E}_{\Lambda} ; \\
\mathscr{E}_{\Lambda} & =\frac{\tau_{\Lambda}}{2 m_{\Lambda}}+\left(\frac{m_{\Lambda}}{m_{\Lambda}^{*}}-1\right) \frac{\tau_{\Lambda}-C \rho_{\Lambda}^{5 / 3}}{2 m_{\Lambda}}+\mathscr{E}_{N \Lambda} ; \\
\mathscr{E}_{N \Lambda} & =\left(\rho_{N}+\rho_{\Lambda}\right) \frac{B}{A}\left(\rho_{N}, \rho_{\Lambda}\right)-\rho_{N} \frac{B}{A}\left(\rho_{N}, 0\right)-\frac{C \rho_{\Lambda}^{5 / 3}}{2 m_{\Lambda}}
\end{aligned}
$$

where the energy density functional $\mathscr{E}_{N \Lambda}$ is obtained from a fit to the binding energy per baryon, $B / A\left(\rho_{N}, \rho_{\Lambda}\right)$ of asymmetric hypermatter, generated by BHF calculations [55]. The adequate $\Lambda$ effective mass $m_{\Lambda}^{*}$ is computed from the BHF single- $\Lambda$ potential obtained in the same calculations. Then the HF equations for a hypernuclei system can be routinely obtained from the total energy functional of Eq. (9), as detailed in Ref. [43, 54].

In this work two kinds of Nijmegen soft-core hyperon potentials are used in BHF calculations for the derivation of effective microscopic lambda-nucleon interactions: one is the NSC97f model [52], another is the recently developed ESC08b model [51]. The ESC08b potential contains no hyperon-hyperon (YY) components, whereas the NSC97f potential comprises the extension to the YY sector based on SU(3) symmetry. That is, the pairing interaction between $\Lambda$ hyperons is considered only in the study with the NSC97f potential. The NSC97f potential in the past has been preferred for the study of $\Lambda$ hypernuclei since it reproduces rather well the available experimental binding data of $\Lambda$ hypernuclei [60]. The ESC08b potential, on the other hand, has been demonstrated to have a slightly more attractive $\Lambda$ potential in nuclear matter, with $-40 \mathrm{MeV}$ [61] for ESC08b and -36 MeV [62] for NSC97f, respectively. One can thus expect that it is harder to evolve deformed $\Lambda$ hypernuclei with the ESC08b, which is confirmed later by the comparison between the NSC97f and ESC08b results. 


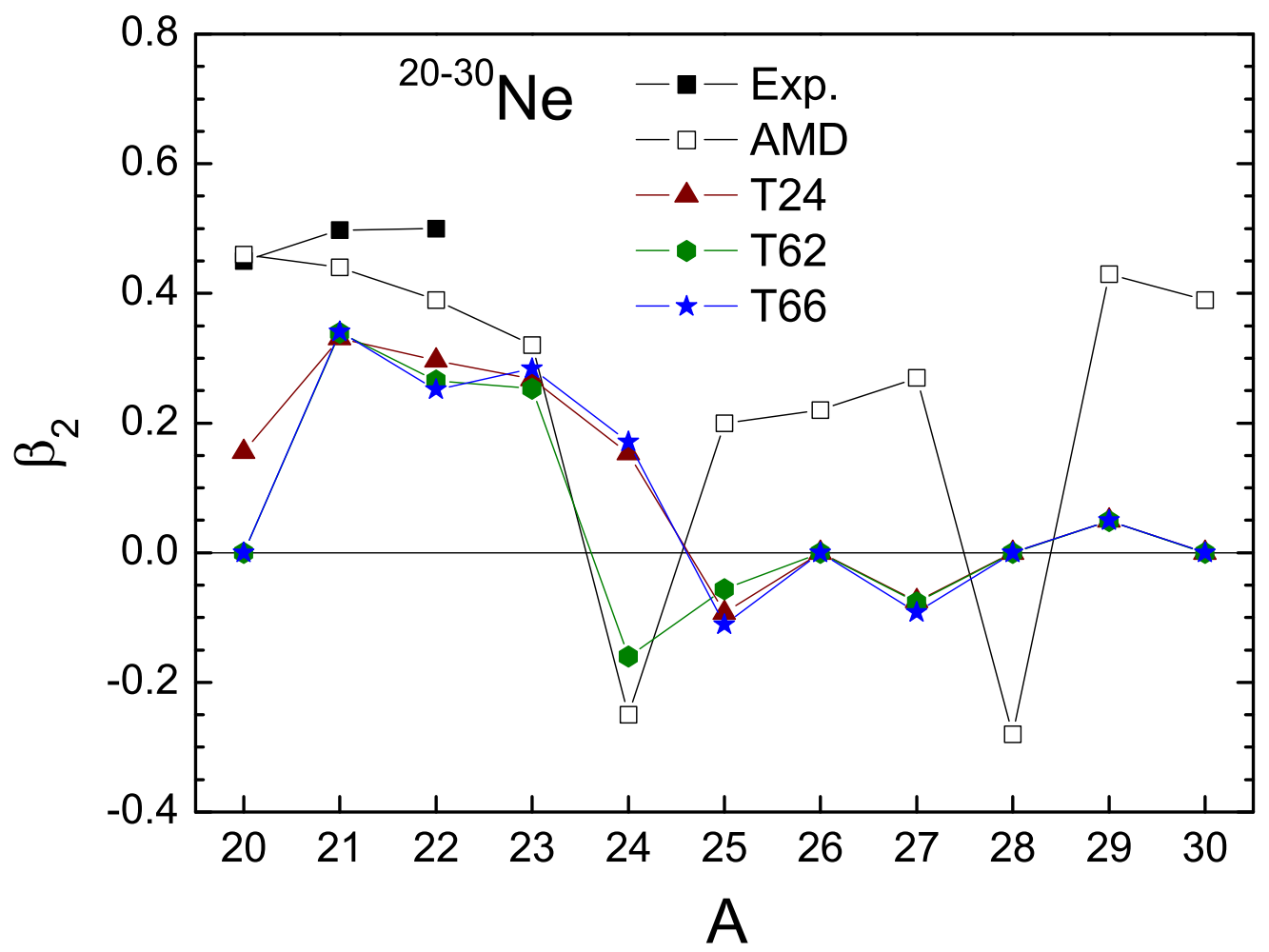

FIG. 1: (Color online) Deformation parameters $\beta_{2}$ of Ne nuclei as a function of the neutron number in comparison with experimental results [1]. The calculated values corresponding to the Skyrme forces T24, T44, T62, T66 [24] effective interactions. The calculated data employing the AMD [11, 12] are also shown for comparison.

\section{RESULTS}

Fig. 1 illustrates the evolution of the axially-symmetric deformations with neutron number along the neon isotopic chain for various effective interactions, in comparison with experimental results [1]. The deformation parameter is defined in the cylindrical coordinate as the expectation values of radius operators $\beta_{2}=\sqrt{\frac{\pi}{5}} \frac{\left\langle 2 z^{2}-r^{2}\right\rangle}{\left\langle z^{2}+r^{2}\right\rangle}$ where the optimal ones are found by minimizing the total energy of the nucleus. If $\beta_{2}>0$ that means the nucleus with a prolate shape while $\beta_{2}<$ 0 means an oblate one. Our selected parameterizations include: The Skyrme force T24 with a substantial like-particle coupling constant $\alpha$ and a vanishing proton-neutron coupling constant $\beta$; T62 with a large proton-neutron coupling constant $\beta$ and a vanishing like-particle coupling constant $\alpha$; and T66 with large and equal proton-neutron and like-particle tensor-term coupling constants. The calculated results of $\mathrm{T} 44$, which has a mixture of like-particle and proton-neutron tensor terms, are found to be practically the same with those of T62 and not shown here in Fig. 1 . The calculated results employing the AMD [11, 12] with a Gogny-D1S interaction are also shown for comparison. Notice that the AMD calculations do not take into account the pairing interaction.

In general, among all the isotopes, the deformations of ${ }^{20} \mathrm{Ne}$ and ${ }^{24} \mathrm{Ne}$ depend much on the interactions adopted. The softness of these nuclei can be understood because they have shallow energy surfaces (shown in Ref. [10] with a different Skyrme interaction SGII), and results are easily changed by a delicate balance among the contributions of energy density functionals in different 

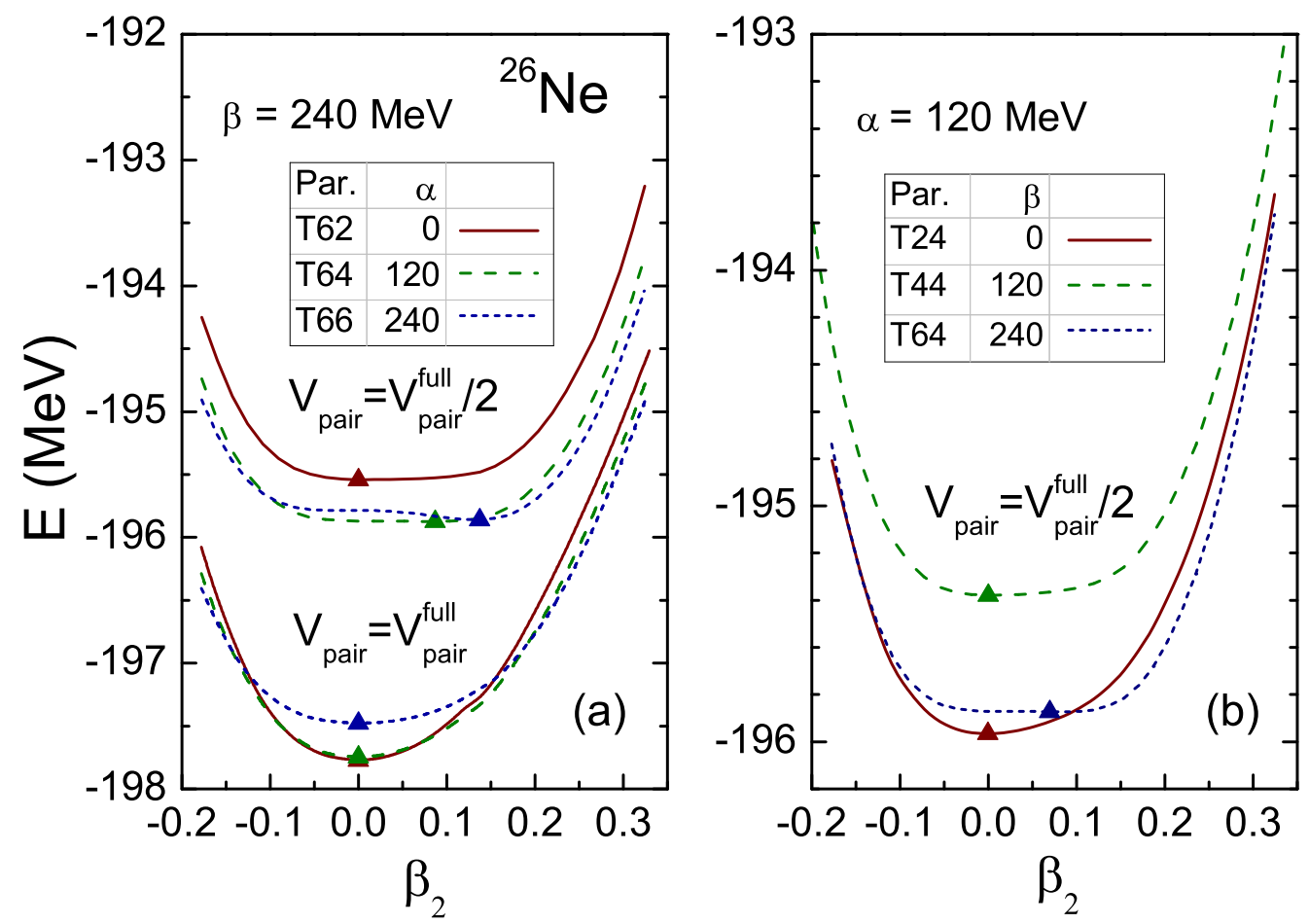

FIG. 2: (Color online) Energy surfaces of ${ }^{26} \mathrm{Ne}$ as a function of the quadruple deformation parameter $\beta_{2}$ using T62 (solid line), T64 (dashed line), T66 (dotted line). The upper three curves are done using a strong full pairing of $V_{\text {pair }}=V_{\text {pair }}^{\text {full }}$, and the lower three curves are with a medium pairing of $V_{\text {pair }}=V_{\text {pair }}^{\text {full }} / 2$. The energy minima are indicated with triangles.

parameterizations. The sensitivity of ${ }^{20} \mathrm{Ne}$ and ${ }^{24} \mathrm{Ne}$ was found also by the relativistic HartreeFock-Bogoliubov (RHFB) model [14]. A decisive theoretical ingredient is the fine structure of the s.p. spectra of those nuclei. Our interest and focus in this work are to understand how spherical shapes of ${ }^{26} \mathrm{Ne}$ and ${ }^{30} \mathrm{Ne}$ are predicted for all of the various parameterizations shown in Fig. 11(The ${ }^{28} \mathrm{Ne}$ nucleus might be triaxially-deformed [11, 12] which is beyond the scope of this work). As mentioned in the introduction, those results are not consistent with the large deformations found in experiments [1-6], and the inconsistency is also present in other theoretical mean-field models such as the Hartree-Fock-Bogoliubov (HFB) theory [13] and the RHFB theory [14]. A better agreement of the AMD results, which include no pairing, suggests the importance of not only pairing correlations on the evolution of deformation, but also extra contributions beyond mean-field models such as the particle-vibration couplings, which needs to be studied in the future. Hereafter, within the present model, we demonstrate how sensitively the shapes of these nuclei depend on the pairing strength together with the cooperative tensor correlation.

In Fig. 2, the energy surfaces of ${ }^{26} \mathrm{Ne}$ are shown as a function of the deformation parameter $\beta_{2}$ with increasing tensor couplings: left panel for increasing values of like-particle coupling $\alpha$ with T62 (solid line), T64 (dashed line), T66 (dotted line) at fixed proton-neutron coupling of $\beta=240 \mathrm{MeV}$, and right panel for increasing proton-neutron coupling $\beta$ with T24 (solid line), T44 (dashed line), T64 (dotted line) at fixed like-particle coupling of $\alpha=120 \mathrm{MeV}$. The calculations are done using both a strong full pairing of $V_{\text {pair }}=V_{\text {pair }}^{\text {full }}$, and a medium pairing of $V_{\text {pair }}=V_{\text {pair }}^{\text {full }} / 2$. The neutron pairing gaps in the latter cases are only a few $\mathrm{keV}$, and are regarded as the cases in which we do one variant of calculations with a weakened pairing to study its influence on the 

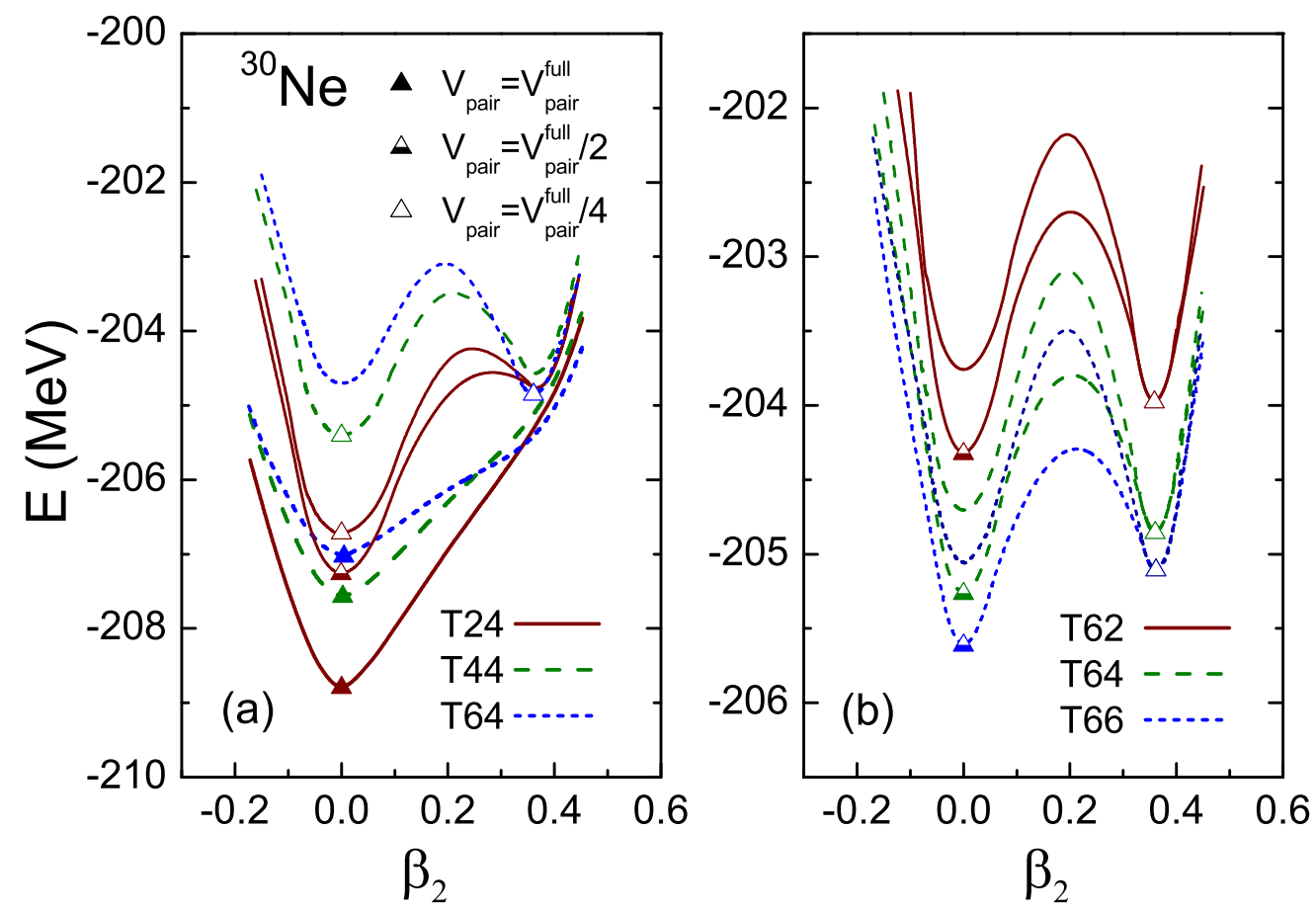

FIG. 3: (Color online) Same with Fig. 2 but for ${ }^{30} \mathrm{Ne}$. The energy minima are indicated with triangles, and three cases of pairing strengths are employed: a strong pairing case with $V_{\text {pair }}=V_{\text {pair }}^{\text {full }}$ (filled symbols), a medium one with $V_{\text {pair }}=V_{\text {pair }}^{\text {full }} / 2$ (half symbols) and a weak one with $V_{\text {pair }}=V_{\text {pair }}^{\text {full }} / 4$ (open symbols). In the left panel, all three pairing cases are shown for T24, and two pairing cases $\left(V_{\text {pair }}=V_{\text {pair }}^{\text {full }}, V_{\text {pair }}^{\text {full }} / 4\right)$ are shown for T44 and T64; In the right panel, two pairing cases $\left(V_{\text {pair }}=V_{\text {pair }}^{\text {full }} / 2, V_{\text {pair }}^{\text {full }} / 4\right)$ are shown for T62, T64 and T66.

nuclear deformation. The energy minima are indicated with triangles. Three cases with $V_{\text {pair }}=$ $V_{\text {pair }}^{\text {full }}$ all give a spherical shape for ${ }^{26} \mathrm{Ne}$, even with increasingly strong tensor forces. While in the case of $V_{\text {pair }}=V_{\text {pair }}^{\text {full }} / 2$, small $\alpha$ value in the case of T62 gives a spherical minima, but larger $\alpha$ values in cases of T64 and T66 drive clear deformations in the prolate side, which indicates an essential role of $\alpha$ tensor strength on the shape of ${ }^{26} \mathrm{Ne}$. Similarly, a larger $\beta$ value results in a prolate deformation, as seen in the right panel of Fig. 2. For example, $\beta_{2}=0.087$ for T64 with a large value of $\beta=240 \mathrm{MeV}$ with $\alpha=120 \mathrm{MeV}$. Therefore, to obtain an experimentally observed prolate shape of ${ }^{26} \mathrm{Ne}$, a relatively large tensor strength is obviously necessary, together with a weakened pairing between nucleons. We mention here that this prolate result was predicted before only by theoretical calculations based on the RHFB model using one parameter set of PKO3 with $\beta_{2} \sim 0.2[14]$.

For the case of ${ }^{30} \mathrm{Ne}$, we prepare in Fig. 3 its energy surfaces using the same parameter sets in Fig. 2. The energy minima are indicated with triangles, and three cases of pairing strengths

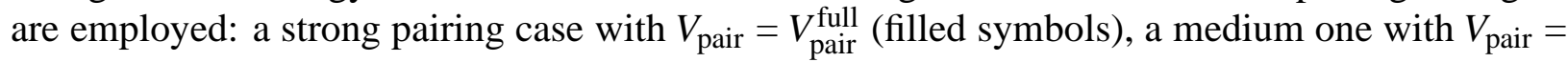
$V_{\text {pair }}^{\text {full }} / 2$ (half symbols) and a weak one with $V_{\text {pair }}=V_{\text {pair }}^{\text {full }} / 4$ (open symbols). From the comparison of the T24 results with those of T44 and T64, we see that a stronger tensor coupling in the selfconsistent SHF calculation makes the energy surface shallower, and at the same time makes the second prolate minimum more pronounced. However, the spherical minimums still win in the case of $V_{\text {pair }}=V_{\text {pair }}^{\text {full }}$ (filled symbols), and in the case of $V_{\text {pair }}=V_{\text {pair }}^{\text {full }} / 2$ (half symbols) as well, even for the 
latter case, a weakened pairing helps to lift largely in energy the spherical minimums. Interesting results are obtained with a further weakened pairing in the case of $V_{\text {pair }}=V_{\text {pair }}^{\text {full }} / 4$ (open symbols). In this circumstance, T24 and T44 with smaller tensor strengthes give still no deformed minima, but T62, T64, T66 with larger tensor couplings finally achieve a large prolate-deformed shape at $\beta_{2} \sim 0.35$ for ${ }^{30} \mathrm{Ne}$, as desired by the experiments. This suggests that it demands the cooperation of a small pairing strength and a large tensor force to obtain a large prolate deformation for ${ }^{30} \mathrm{Ne}$ in this SHF + BCS model. This is consistent with the conclusion drawn in the context of ${ }^{26} \mathrm{Ne}$.

Our results demonstrate very clearly that the nucleon pairing together with the tensor correlation are responsible to evolve the shape of nuclei. The decisive role of a weak nucleon pairing can be understood from a well-known fact that the pairing interaction tends to form the $J=0^{+}$ pairs of identical particles which have spherically symmetric wave functions. The appearance of well-deformed local minima in the weak pairing cases may be indicated in the corresponding s.p. configurations at the minima. To take T64, as an example, [330 $1 / 2]$ level from $1 f_{7 / 2}$ orbit is occupied with the occupation probability $v^{2}=1.0$, while [202 3/2] level from $1 d_{3 / 2}$ orbit is unoccupied with $v^{2}=0.0$. Here one-particle levels are given in the standard notation of asymptotic quantum numbers $\left[N n_{z} \Lambda \Omega\right]$. Those configurations largely differ in the strong pairing case, where the corresponding occupation probability for $1 f_{7 / 2}$ orbit is 0.05 , and that for $1 d_{3 / 2}$ orbit is 0.90 . Meanwhile, the importance of tensor interaction for generating deformed minima is due to the fact that the tensor interaction brings in general reduced neutron and proton shell gaps and enhanced s.p. level densities near the Fermi level. To take T24 and T64 as a comparison, the $1 f_{7 / 2}-1 d_{3 / 2}$ neutron gap is reduced from $4.49 \mathrm{MeV}$ to $3.76 \mathrm{MeV}$, and the $1 d_{5 / 2}-1 p_{1 / 2}$ proton gap is reduced from $8.82 \mathrm{MeV}$ to $7.73 \mathrm{MeV}$.

In order to illustrate more clearly the shape change due to the tensor force in ${ }^{26} \mathrm{Ne}$, we present in Fig. 4 a correlation between tensor coupling strength $\alpha$ or $\beta$ and deformation $\beta_{2}$ at the energy minimum of ${ }^{26} \mathrm{Ne}$ at fixed $\beta=240 \mathrm{MeV}$ (left panel) and $\alpha=120 \mathrm{MeV}$ (right panel), in the case of a medium pairing with $V_{\text {pair }}=V_{\text {pair }}^{\text {full }} / 2$. The corresponding singe- and double-hypernuclei are also presented with two hyperon interactions: the ESC08b model (filled symbols) and the NSC97f model (open symbols). In general, the addition of the $\Lambda$ particle results in a slightly smaller deformation, as is the same with the earlier study [43] with the absence of the tensor force. And from Fig.4(a) we notice that with the ESC08b model hypernuclei tend to be more spherical, which has its root in a deeper $\Lambda$ potential for ESC08b than for NSC97f as mentioned before. Specially, due to the weak deformation minimum in this medium pairing case, the double- $\Lambda$ hypernuclei ${ }_{\Lambda \Lambda}^{28} \mathrm{Ne}$ is found to be spherical when including the contribution of strong YN interaction of ESC08b, compared with a prolate ${ }^{26} \mathrm{Ne}$ core.

Our final results are presented in Fig. 5, where the deformation parameters of the doublelambda hypernuclei (open symbols) with neon isotopic core ${ }^{20-30} \mathrm{Ne}$ are plotted using the ESC08b potential, with the comparison of the data of those of core nuclei (filled symbols). The calculations are done with T66 for two cases of pairing strengths: a full pairing of $V_{\text {pair }}=V_{\text {pair }}^{\text {full }}$, and a weak pairing of $V_{\text {pair }}=V_{\text {pair }}^{\text {full }} / 4$. The softness of ${ }^{20,24} \mathrm{Ne}$ is again present, and we also find an interesting shape inverse of ${ }^{25} \mathrm{Ne}$ from oblate to prolate with the modification of pairing strength. And prolately-deformed ground states are successfully realized for ${ }^{26,28,30} \mathrm{Ne}$ as a combined effect of a large tensor force and a weakened pairing in the present model. This fact might be quite meaningful for further improvements of the SHF model or Skyrme parameterizations toward a better description on the shell structures of nuclei in general. As was stated before, we see that for all the isotopes there are smaller deformations with the same shapes for hypernuclei, compared with corresponding core nuclei in both the full pairing and the weak pairing cases. 

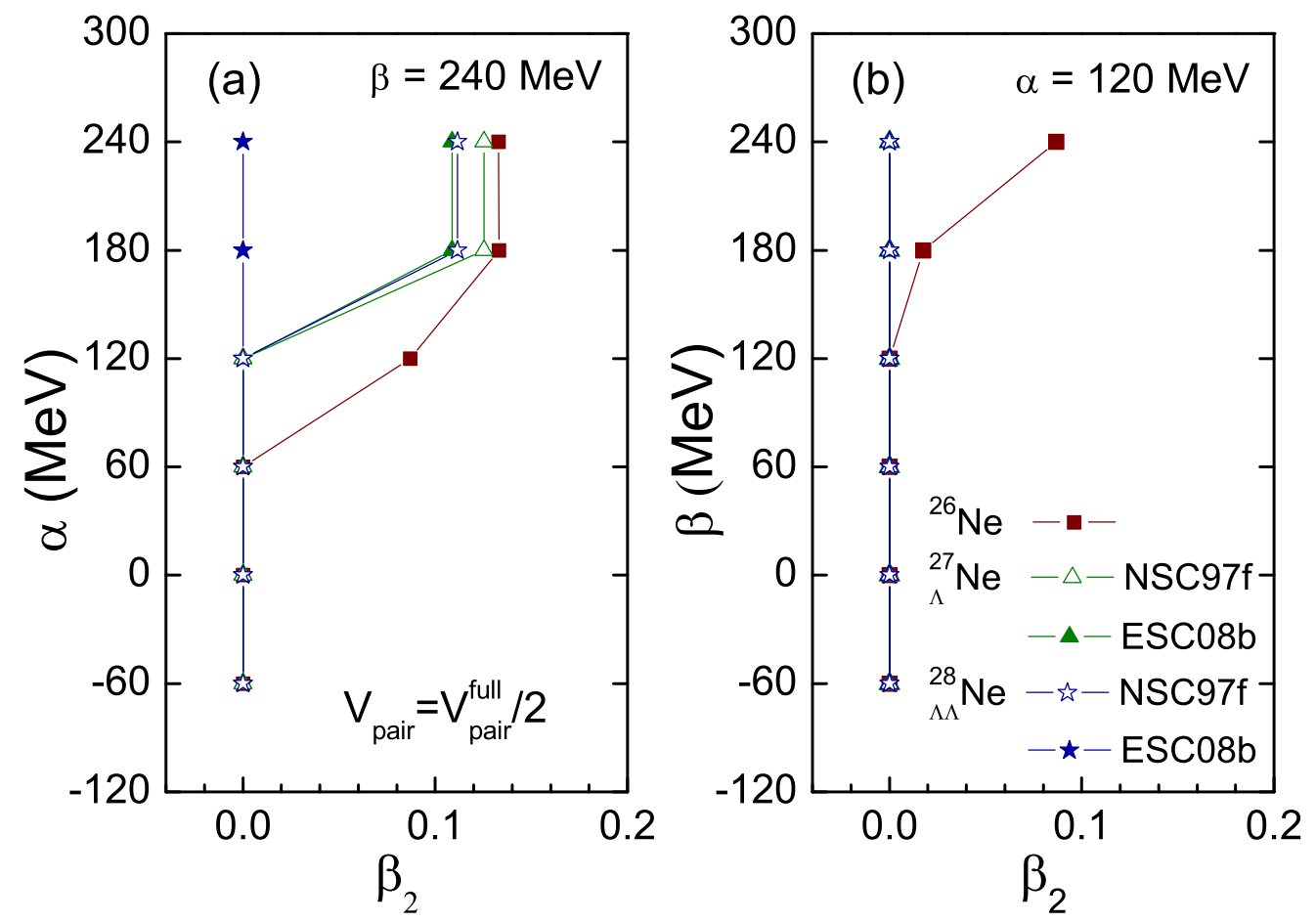

FIG. 4: (Color online) Left panel: Deformation changing of ${ }^{26} \mathrm{Ne}$ with increasing tensor coupling strength $\alpha$ using T61, T62, T63, T64, T65, T66, for $\beta=240 \mathrm{MeV}$; Right panel: Deformation changing of ${ }^{25} \mathrm{Ne}$ with increasing tensor coupling strength $\beta$ using T14, T24, T34, T44, T54, T64, for $\alpha=120 \mathrm{MeV}$. The calculations are done with a medium pairing of $V_{\text {pair }}=V_{\text {pair }}^{\text {full }} / 2$. The corresponding singe- and doublehypernuclei are also presented in dashed and dotted lines with two hyperon interactions: the ESC08b model (filled symbols) and the NSC97f model (open symbols).

\section{SUMMARY AND FUTURE PERSPECTIVES}

In summary, we have performed the deformed SHF + BCS model calculations to investigate the effects of tensor and pairing forces on the quadruple deformation of neon isotopes and the corresponding $\Lambda$ hypernuclei. With selected parameterizations of various tensor, pairing and hyperonnucleon interactions, we disentangle the interplay of these correlations for the deformation of neon isotopes and the corresponding hypernuclei. To investigate the role of the pairing correlations, we adopt three kinds of the pairing strength. For tensor interactions, we take 11 different Skyrme parameter sets listed in Table 1. With these parameter sets, we found in ${ }^{25} \mathrm{Ne}$ and ${ }^{26} \mathrm{Ne}$ the important interplay of the tensor and pairing correlations which are different to those in the lighter neon isotopes. Namely, in ${ }^{21,22,23} \mathrm{Ne}$, the ground states are predicted always to be prolate deformed, irrespective to the adopted interaction, which are consistent with both experiments and previous calculations. The increase of tensor strength changes the shape of ${ }^{25} \mathrm{Ne}$ nucleus from oblate to prolate, and also the shape of ${ }^{26} \mathrm{Ne}$ nucleus from spherical to prolate with the help of a weakened nucleon pairing interaction. The prolate shape of ${ }^{26} \mathrm{Ne}$ obtained with relatively large tensor strengths is quite encouraging because it is consistent with the experimental $B(E 2)$ data. We demonstrate also that the cooperation of a weakened pairing and a large tensor interaction drives the shape of ${ }^{30} \mathrm{Ne}$ from spherical to prolate, as desired by the recent experiments.

In addition, the interplay of tensor force and hyperon force is also studied, and the tensor 


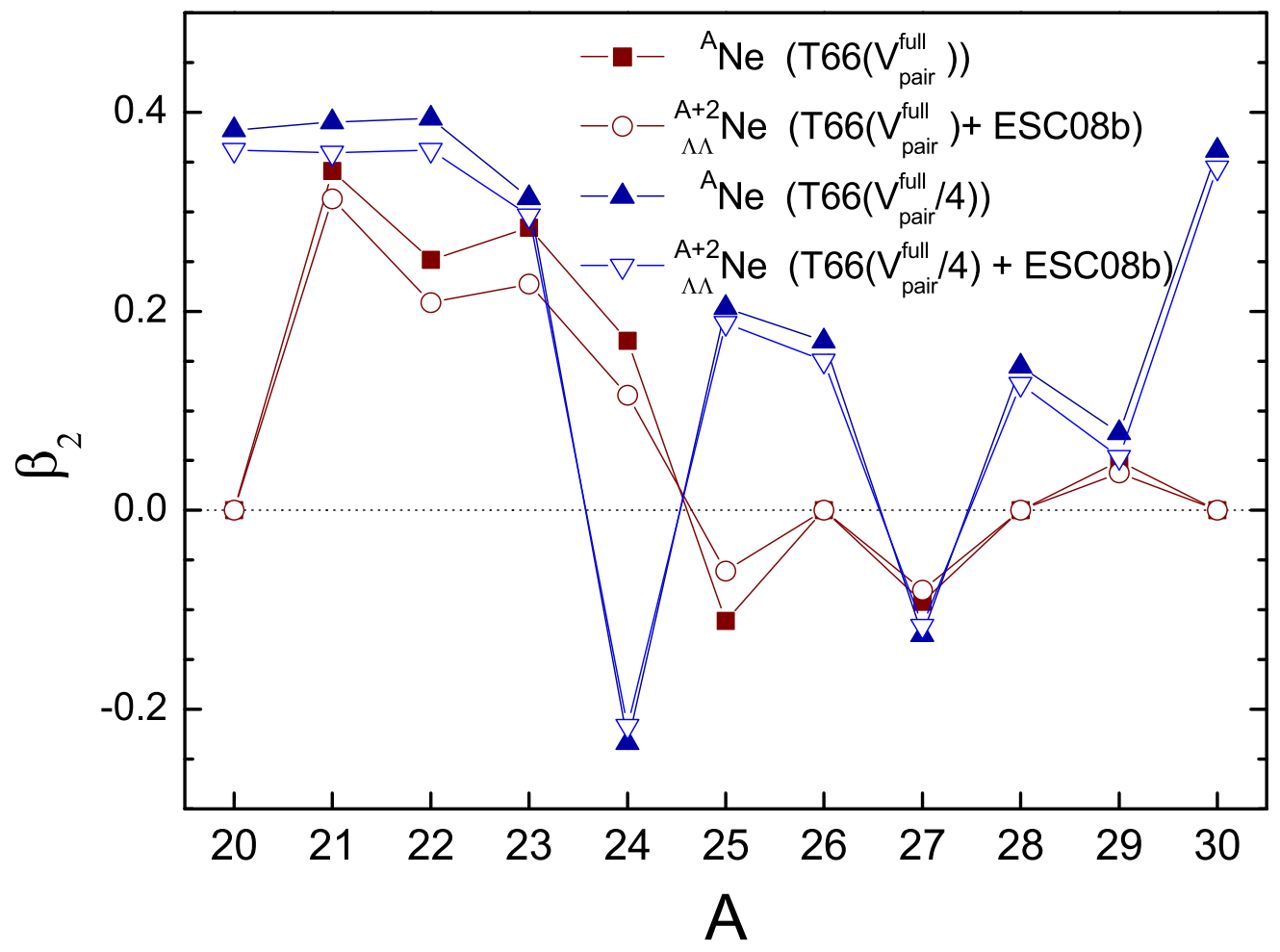

FIG. 5: (Color online) Deformation parameters of the double-lambda hypernuclei (filled symbols) with neon isotopic core ${ }^{20-30} \mathrm{Ne}$ are plotted using the ESC08b potential, with the comparison of those of core nuclei (open symbols). The calculations are done with T66 for two cases of pairing strengths: a full pairing of $V_{\text {pair }}=V_{\text {pair }}^{\text {full }}$, and a weak pairing of $V_{\text {pair }}=V_{\text {pair }}^{\text {full }} / 4$.

effect on the deformation of the isotope is found to be larger than that of $\Lambda$ particles added to the core nucleus with realistic hyperon interactions. With the same core nuclei, the ESC08b potential makes the corresponding single- and double-hypernuclei harder to deform than the NSC97f model because of a deeper $\Lambda$ potential depth of the former case.

As a future project, it is quite important to examine further the strengths of the realistic tensor interaction for the mean field models, for which we may have to refer to more microscopic calculations of the nuclear energy density functional based on realistic NN interactions, such as chiral NN potential N3LOW [63, 64]. With that our calculation might be improved using a microscopicdetermined tensor strength parameters (which might be density-dependent) with more insight into the tensor role on nuclear structures. Also, to clarify the pairing effect, as mentioned in the introduction, we should update our calculation with a recently proposed isospin-dependent pairing force, using a proper pairing strength parameters fitted from the systematic experimental pairing gaps [37, 65]. At the same time, it might be important to accommodate the effects beyond the mean-field model such as the particle-vibration coupling effect in the future study. 


\section{Acknowledgments}

We would like to thank Prof. J. Meng, Prof. K. Hagino and Dr. H.Z. Liang for valuable discussions. This work is supported by a Grants-in-Aid for Scientific Research from Monbukagakusho of Japan (21540288 and 20105003), and by the National Natural Science Foundation of China (Grant No 10905048, 10975116 and 11275160).

[1] S. Raman, C. W. Nestor Jr., and P. Tikkanen, At. Data Nucl. Data Tables 78, 1 (2001).

[2] L. A. Riley et al., Phys. Rev. C 68, 044309 (2003).

[3] Y. Yanagisawa at al., Phys. Lett. B 566, 84 (2003).

[4] W. Geithner et al., Phys. Rev. C 71, 064319 (2005).

[5] H. Iwasaki et al., Phys. Lett. B 620, 118 (2005).

[6] J. Gibelin et al. Phys. Rev. C 75, 057306 (2007).

[7] M. Takechi et al., Phys. Lett. B 707, 357 (2012).

[8] T. Nakamura et al., Phys. Rev. Lett. 103, 262501 (2009).

[9] Y. Urata, K. Hagino, and H. Sagawa, Phys. Rev. C 83, 041303(R) (2011).

[10] H. Sagawa, X. R. Zhou, X. Z. Zhang, and T. Suzuki, Phys. Rev. C 70, 054316 (2004).

[11] K. Minomo, T. Sumi, M. Kimura, K. Ogata, Y. R. Shimizu, and M. Yahiro, Phys. Rev. Lett. 108, 052503 (2012).

[12] T. Sumi, K. Minomo, S. Tagami, M. Kimura, T. Matsumoto, K. Ogata, Y. R. Shimizu, and M. Yahiro, Phys. Rev. C 85, 064613 (2012).

[13] S. Hilaire and M. Girod, Eur. Phys. J. A 33, 237 (2007).

[14] J.-P. Ebran, E. Khan, D. Pena Arteaga, and D. Vretenar, Phys. Rev. C 83, 064323 (2011).

[15] T. Otsuka, T. Suzuki, and Y. Utsuno, Nucl. Phys. A 805, 127 (2008).

[16] Y. Utsuno, T. Otsuka, B. A. Brown, M. Honma, and T. Mizusaki, AIP Conf. Proc. 1120, 81 (2009).

[17] Y. Utsuno, T. Otsuka, B. A. Brown, M. Honma, T. Mizusaki, and N. Shimizu, arXiv:1201.4077

[18] T. Otsuka, T. Suzuki, R. Fujimoto, H. Grawe, and Y. Akaishi, Phys. Rev. Lett. 95, 232502 (2005).

[19] T. Otsuka, R. Fujimoto, Y. Utsuno, B. A. Brown, M. Honma, and T. Mizusaki, Phys. Rev. Lett. 87 $082502(2001)$

[20] T. H. R. Skyrme, Phil. Mag. 1, 1043 (1956); J. S. Bell and T. H. R. Skyrme, ibid. 1, 1055 (1956); T. H. R. Skyrme, Nucl. Phys. 9, 615 (1958); T. H. R. Skyrme, ibid. 9, 635 (1958).

[21] B. A. Brown, T. Duguet, T. Otsuka, D. Abe, T. Suzuki, Phys. Rev. C 74, 061303 (2006).

[22] G. Colò, H. Sagawa, S. Fracasso, and P. F. Bortignon, Phys. Lett. B 646, 227 (2007).

[23] D. M. Brink and FI. Stancu, Phys. Rev. C 75, 064311 (2007).

[24] T. Lesinski, M. Bender, K. Bennaceur, T. Duguet, and J. Meyer, Phys. Rev. C 76, 014312 (2007) and references therein.

[25] M. Grasso, Z. Y. Ma, E. Khan, J. Margueron, and N. Van Giai, Phys. Rev. C 76, 044319 (2007).

[26] W. Zou, G. Colò, Z. Y. Ma, H. Sagawa, and P. F. Bortignon, Phys. Rev. C 77, 014314 (2008).

[27] M. Zalewski, J. Dobaczewski, W. Satula, and T. R. Werner, Phys. Rev. C 77, 024316 (2008).

[28] D. Tarpanov, H. Z. Liang, N. V. Giai, and C. Stoyanov, Phys. Rev. C 77, 054316 (2008).

[29] C. L. Bai, H. Sagawa, H. Q. Zhang, X. Z. Zhang, G. Colò, and F. R. Xu, Phys. Lett. B 675, 28 (2009).

[30] C. L. Bai, H. Q. Zhang, X. Z. Zhang, F. R. Xu, H. Sagawa, and G. Colò, Phys. Rev. C 79, 041301(R) (2009).

[31] L. G. Cao, G. Colò, H. Sagawa, P. F.Bortignon, and L. Sciacchitano, Phys. Rev. C 80, 064304 (2009). 
[32] M. Moreno-Torres, M. Grasso, H. Liang, V. DeDonno, M. Anguiano, and N. VanGiai, Phys. Rev. C 81, 064327 (2010).

[33] C. L. Bai, H. Q. Zhang, H. Sagawa, X. Z. Zhang, G. Colò, and F. R. Xu, Phys. Rev. Lett. 105, 072501 (2010).

[34] C. L. Bai, H. Q. Zhang, H. Sagawa, X. Z. Zhang, G. Colò, and F. R. Xu, Phys. Rev. C 83, 054316 (2011).

[35] L. G. Cao, H. Sagawa, and G. Colò, Phys. Rev. C 83, 034324 (2011).

[36] C. L. Bai, H. Sagawa, G. Colò, H. Q. Zhang, and X. Z. Zhang, Phys. Rev. C 84, 044329 (2011).

[37] J. Margueron, H. Sagawa, and K. Hagino, Phys. Rev. C 76, 064316 (2007).

[38] J. Margueron, H. Sagawa, and K. Hagino, Phys. Rev. C 77, 054309 (2008).

[39] C. A. Bertulani, Hongliang Liu, and H. Sagawa, Phys. Rev. C 85, 014321 (2012).

[40] T. Motoba, H. Bandō, and K. Ikeda, Prog. Theor. Phys. 70, 189 (1983); T. Motoba, H. Bandō, K. Ikeda, and T. Yamada, Prog. Theor. Phys. Suppl. 81, 42 (1985).

[41] E. Hiyama, M. Kamimura, K. Miyazaki and T. Motoba, Phys. Rev. C 59, 2351 (1999).

[42] K. Tanida et al., Phys. Rev. Lett. 86 (2001), 1982.

[43] X. R. Zhou, H.-J. Schulze, H. Sagawa, C. X. Wu, and E. G. Zhao, Phys. Rev. C 76, 034312 (2007).

[44] M. T. Win and K. Hagino, Phys. Rev. C 78, 054311 (2008).

[45] B. N. Lu, E. G. Zhao, and S. G. Zhou, Phys. Rev. C 84, 014328 (2011).

[46] H.-J. Schulze, M. T. Win, K. Hagino, H. Sagawa, Prog. Theor. Phys. (Kyoto) 123, 569 (2010).

[47] M. Isaka, M. Kimura, A. Dote, and A. Ohnishi, Phys. Rev. C 83, 044323 (2011).

[48] D. J. Millener, Lecture Notes in Phys. 31, 724 (2007) ; Nucl. Phys. A 804, 84 (2008).

[49] E. Hiyama, M. Kamimura, T. Motoba, T. Yamada and Y. Yamamoto, Phys. Rev. Lett. 85, 270 (2000).

[50] E. Hiyama, Y. Yamamoto, Th. A. Rijken and T. Motoba, Phys. Rev. C 74, 054312 (2006).

[51] T. Rijken, M. Nagels, and Y. Yamamoto, Nucl. Phys. A 835, 160 (2010); Y. Yamamoto, E. Hiyama, and T. Rijken, ibid. 835, 350 (2010); T. Rijken, M. Nagels, and Y. Yamamoto, Prog. Theor. Phys. Suppl. 185, 14 (2010); Y. Yamamoto, T. Motoba, and T. Rijken, ibid. 185, 72 (2010).

[52] V. G. J. Stoks and Th. A. Rijken, Phys. Rev. C 59, 3009 (1999).

[53] D. Vautherin, Phys. Rev. C 7, 296 (1973).

[54] J. Cugnon, A. Lejeune, and H.-J. Schulze, Phys. Rev. C 62, 064308 (2000).

[55] H.-J. Schulze, A. Lejeune, J. Cugnon, M. Baldo, and U. Lombardo, Phys. Lett. B 355, 21 (1995); H.-J. Schulze, M. Baldo, U. Lombardo, J. Cugnon, and A. Lejeune, Phys. Rev. C 57, 704 (1998); M. Baldo, G. F. Burgio, H.-J. Schulze, Phys. Rev. C 61, 055801 (2000); H.-J. Schulze, A. Polls, A. Ramos, and I. Vidaña, Phys. Rev. C 73, 058801 (2006).

[56] P.-G Reinhard and H. Flocard, Nucl. Phys. A 584, 467 (1995).

[57] W. Satula, J. Dobaczewski, and W. Nazarewicz, Phys. Rev. Lett. 81, 3599 (1998).

[58] G. Audi, A. H. Wapstra, and C. Thibault, Nucl. Phys. A 729, 337 (2003).

[59] M. Bender, K. Rutz, P.-G. Reinhard, and J.A. Maruhn, Eur. Phys. J. A 8, 59 (2000).

[60] Y. Yamamoto, private comunication.

[61] H.-J. Schulze and T. Rijken, Phys. Rev. C 84, 035801 (2011).

[62] I. Vidaña, A. Polls, A. Ramos, and H.-J. Schulze, Phys. Rev. C 64, 044301 (2001).

[63] J. W. Holt, N. Kaisera, and W. Weise, Eur. Phys. J. A 47, 128 (2011).

[64] N. Kaiser, Eur. Phys. J. A 48, 36 (2012).

[65] M. Yamagami, J. Margueron, H. Sagawa, and K. Hagino, Phys. Rev. C 86, 034333 (2012). 